\title{
Focal Encephalitis, Meningitis, and Acute Respiratory Distress Syndrome Associated with Influenza A Infection
}

\author{
Chih-Yu Lianga, b Chih-Hui Yang ${ }^{c}$ Jiun-Nong Lind, e
}

${ }^{a}$ Department of Emergency Medicine, E-Da Cancer Hospital, I-Shou University, Kaohsiung, Taiwan; ${ }^{b}$ Department of Information Engineering, I-Shou University, Kaohsiung, Taiwan; ' Department of Biological Science and Technology,

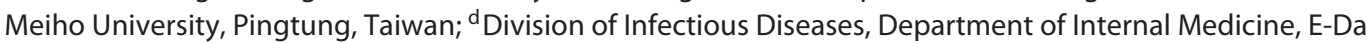

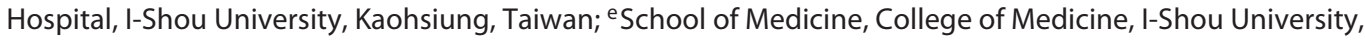
Kaohsiung, Taiwan

\section{Significance of the Study}

- Focal encephalitis is a rare complication following influenza infection. A unique focal T2 prolongation in the left frontoparietal subcortical white matter, without obvious abnormal enhancement or restricted diffusion, was recognized in the brain magnetic resonance imaging scans of this patient.

\section{Keywords}

Influenza infection · Focal encephalitis · Encephalopathy · Meningitis · Seizure · Acute respiratory distress syndrome

\begin{abstract}
Objective: To present a case of influenza A infection complicated with focal encephalitis, meningitis, and acute respiratory distress syndrome. Clinical Presentation and Intervention: A 35-year-old woman presented with fever, headache, cough, and body aches. Seizures, altered consciousness, and dyspnea occurred later. A nasopharyngeal swab revealed a positive reaction for the influenza $A$ antigen. Magnetic resonance imaging scans showed a T2 prolongation in the left frontoparietal subcortical white matter, which was consistent with focal encephalitis. She recovered after treatment with oseltamivir and antibiotics. Conclusion: This case report highlights focal encephalitis with concomitant pulmonary complications after influenza $A$ infection.
\end{abstract}

(c) 2018 The Author(s)

Published by S. Karger AG, Basel

\section{KARGER}

E-Mail karger@karger.com www.karger.com/mpp

\section{(C) 2018 The Author(s)}

Published by S. Karger AG, Basel

Karger

Open access

This is an Open Access article licensed under the Creative Commons Attribution-NonCommercial-4.0 International License (CC BY-NC) (http://www.karger.com/Services/OpenAccessLicense), applicable to the online version of the article only. Usage and distribution for commercial purposes requires written permission.

\section{Introduction}

Influenza is a common infectious disease worldwide and it can easily spread from person to person. It is estimated that influenza annually infects $5-10 \%$ of adults and $20-30 \%$ of children, resulting in approximately $3-5$ million cases of severe illness and 250,000-500,000 deaths globally every year [1]. The severe sequelae of influenza infection include pneumonia, myocarditis, and neurologic manifestations [2-4]. The most common neurologic manifestations of influenza infections include seizures, meningitis, encephalopathy, encephalitis, Reye syndrome, myelitis, movement disorders, and Guillain-Barré syndrome [2-4]. A study in the USA reported an incidence of severe influenza-associated neurologic complications in 1.2 cases per 100,000 persons during the pandemic influenza A (H1N1) of 2009 [4]. In this case report, we describe an adult female with influenza $\mathrm{A}$ infection presenting with acute respiratory distress syndrome, meningoencephalitis, and encephalopathy. A

Jiun-Nong Lin

Division of Infectious Diseases, Department of Internal Medicine, E-Da Hospital, I-Shou University, No. 1, Yida Road, Jiaosu Village, Yanchao District

Kaohsiung 824 (Taiwan)

E-Mail jinoli@kmu.edu.tw 

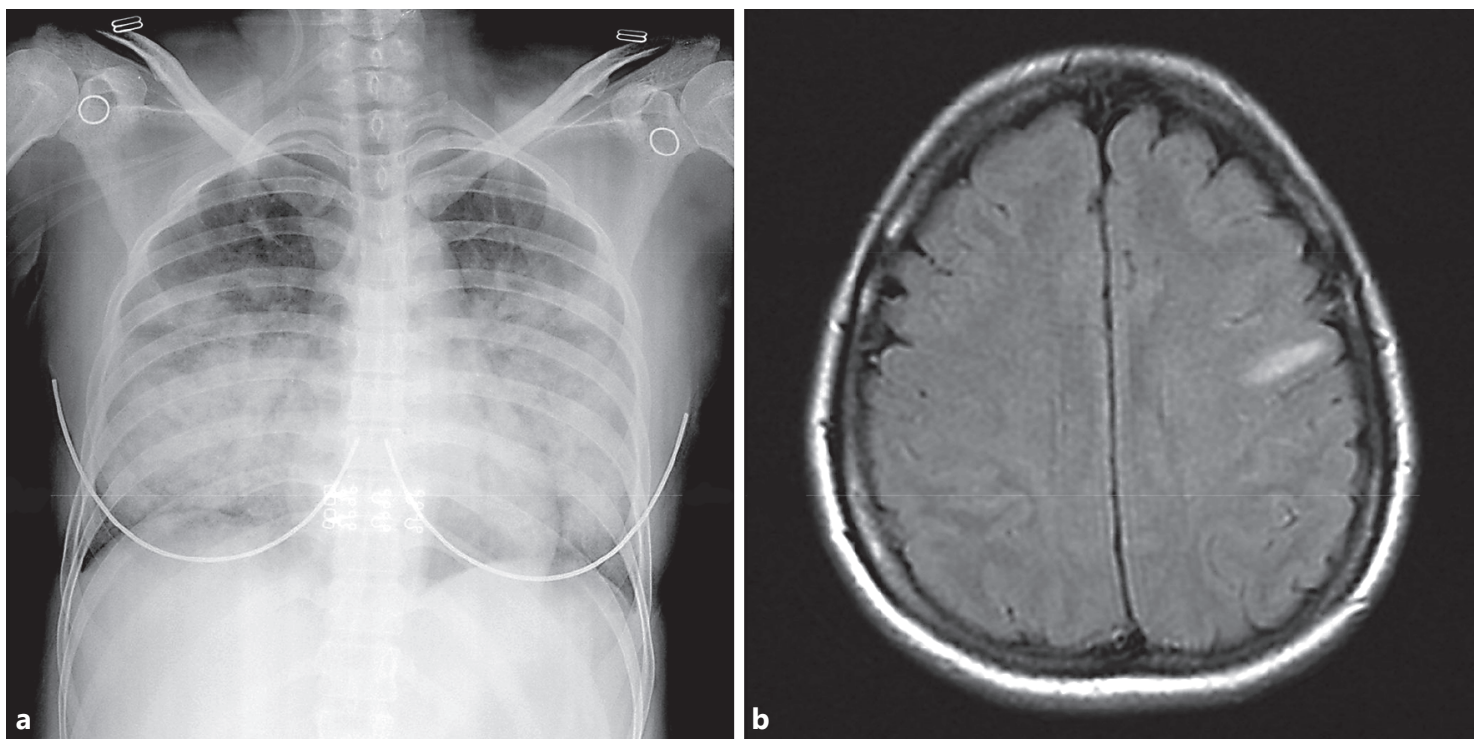

Fig. 1. Chest X-ray and magnetic resonance imaging of the brain. a Severe interstitial infiltration in both lung fields. b Single focal T2 prolongation in the left frontoparietal subcortical white matter.

unique lesion of focal encephalitis was identified from the magnetic resonance imaging (MRI) scans of her brain.

\section{Case Report}

A 35-year-old woman sought care at a community hospital for fever, headache, mild cough, whole body aches, and fatigue lasting 6 days. She had sicca syndrome and had received hydroxychloroquine treatment for 4 years. The rapid influenza antigen test on nasopharyngeal swab revealed a positive reaction for influenza $\mathrm{A}$ and a negative reaction for influenza B. Oseltamivir $(75 \mathrm{mg}$ orally twice a day) was prescribed based on the presumptive diagnosis of influenza A infection. However, progressive deterioration of her clinical condition, including seizures, altered consciousness, and dyspnea, occurred 3 days later. She was then referred to our hospital.

On arrival at our hospital, the patient was in respiratory distress and she was stuporous (Glasgow coma scale, E4V1M3). No other seizures were observed. Her vital signs were as follows: blood pressure, $117 / 76 \mathrm{~mm} \mathrm{Hg}$; pulse rate, 87 beats/min; respiratory rate, 36 breaths/min; and body temperature, $36.8^{\circ} \mathrm{C}$. Physical examination revealed neck stiffness and inspiratory crackles in both lungs. Her chest X-ray showed interstitial infiltration of both lungs (Fig. 1a). Peripheral blood tests revealed a white blood cell count of $6,060 / \mathrm{mm}^{3}$, with $78 \%$ neutrophils and $17 \%$ lymphocytes; hemoglobin, $13.4 \mathrm{~g} / \mathrm{dL}$; and platelets, $153,000 / \mathrm{mm}^{3}$. The results of routine electrolyte tests were normal. A serologic test for human immunodeficiency virus was negative, as were urine antigen tests for Streptococcus pneumoniae and Legionella pneumophila. Arte- rial blood gas analysis showed: $\mathrm{pH}, 7.386 ; \mathrm{PaO}_{2}, 47.9 \mathrm{~mm} \mathrm{Hg}$; $\mathrm{PaCO}_{2}, 44.3 \mathrm{~mm} \mathrm{Hg} ; \mathrm{HCO}_{3}, 26 \mathrm{mM} / \mathrm{L}$; and oxygen saturation, $83 \%$. A nonrebreathing mask with an oxygen delivery of $12 \mathrm{~L} / \mathrm{min}$ was used for her respiratory distress. The computed tomography (CT) scans of her brain were unremarkable, but the MRI scans showed a single focal T2 prolongation in the left frontoparietal subcortical white matter without obvious abnormal enhancement or restricted diffusion (Fig. 1b). This finding was consistent with focal encephalitis. A lumbar puncture was performed; analysis of the cerebrospinal fluid (CSF) revealed the following: opening pressure, $300 \mathrm{~mm} \mathrm{H}_{2} \mathrm{O}$; white blood cell count, 7 cells $/ \mathrm{mm}^{3}$, with $82 \%$ lymphocytes; red blood cell count, $1 \mathrm{cell} / \mathrm{mm}^{3}$; glucose, 60 $\mathrm{mg} / \mathrm{dL}$ (serum, $109 \mathrm{mg} / \mathrm{dL}$ ); and total protein, $786.1 \mathrm{mg} / \mathrm{dL}$. The following tests were negative: Gram staining, acid-fast staining, India ink staining, herpes simplex virus immunoglobulin $\mathrm{M}$, cultures for bacteria, tuberculosis, fungi, and viruses, polymerase chain reaction (PCR) for mycobacterium, and reverse transcription PCR (RT-PCR) for the influenza virus. The influenza viral culture and nasopharyngeal swab RT-PCR, which were obtained 9 days after the onset of symptoms and 3 days after the treatment of oseltamivir, were also negative.

Oral oseltamivir was continued after admission, and intravenous vancomycin and ceftazidime were also administered. Her consciousness returned to a normal level gradually, and her respiratory pattern became even after treatment. A chest X-ray performed 5 days after admission showed a significant reduction of the bilateral lung infiltration (Fig. 2a). She was discharged after 15 days and fully recovered clear consciousness and normal respiratory function. The focal T2 prolongation lesion of the left frontoparietal subcortical white matter had disappeared in the follow-up brain MRI scans obtained 2 months later (Fig. 2b). 

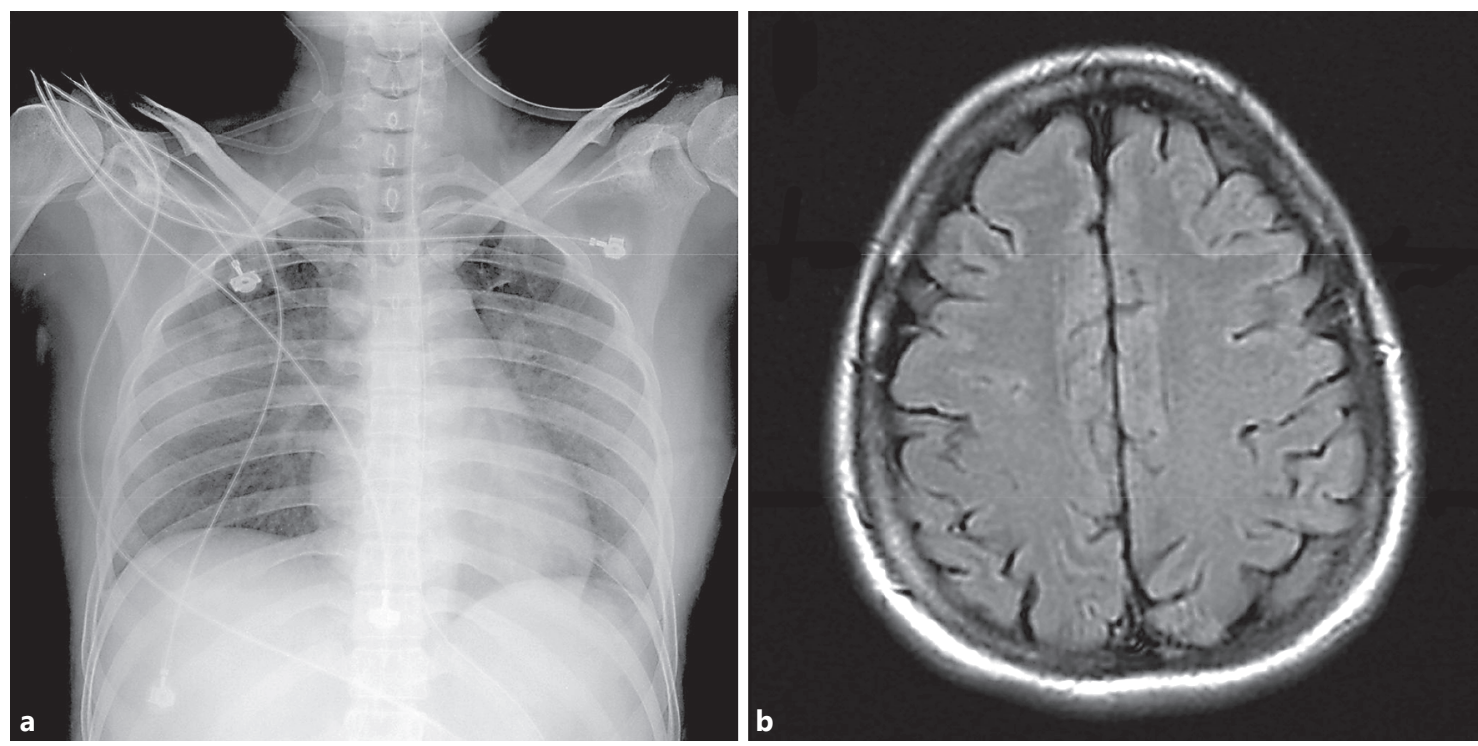

Fig. 2. Follow-up chest X-ray and magnetic resonance imaging of the brain. a Improvement of the infiltration in both lungs 5 days after admission. b Disappearance of the previous focal T2 prolongation lesion in the left frontoparietal subcortical white matter 2 months after admission.

\section{Discussion}

A number of pulmonary and central nervous system complications of influenza injection have been described [2-7], but the focal lesions of T2 prolongation in the subcortical white matter in the MRI scans of the present case have been rarely described. A study conducted in the UK showed that brain CT scans were normal in most patients with influenza-related encephalitis/encephalopathy [3]. In one study performed in Japan during the influenza A (H1N1) pandemic of 2009, 32\% of the patients (31/97) with influenza-related encephalitis/encephalopathy had abnormal brain MRI imaging, including diffuse brain edema, splenial lesions, and abnormal signals in the deep gray matter, the subcortical white matter, and the cortical area [8]. Another smaller study performed in the UK during 2011-2013 showed abnormal brain MRI findings in $18 \%(3 / 17)$ of patients with influenza-associated encephalitis/encephalopathy, including bilateral T2 hyperintensity in the pons, posteromedial thalami, and the right external capsule, as well as cerebellar tonsillar herniation with ischemia at the craniocervical junction [3].

The presentations of influenza-associated neurologic complications are diverse. Seizures are the most common $(57.1 \%)$, followed by encephalopathy/encephalitis (37.7\%), meningitis (3.9\%), and Guillain-Barré syndrome
(1.3\%), as observed in a study conducted in the USA [4]. The outcomes of patients with influenza-associated neurologic complications were diverse in different surveillance studies. The UK surveillance study indicated that most patients (68\%) had poor outcomes and 16\% died, particularly those with encephalopathy/encephalitis [3]. However, the US study reported that most patients had a relatively benign course and that the mortality rate was $5.2 \%$ in patients with influenza-associated neurologic complications [4].

The pathogenesis of influenza-associated encephalitis/ encephalopathy is not entirely clear. Several studies reported that most patients did not have lymphocytic pleocytosis and that the influenza virus was not detected in their CSF [2-7], suggesting that the influenza virus might not directly invade the central nervous system. Instead, the central nervous system manifestations may be secondary to an increased release of cytokines in the brain due to the inflammatory response following influenza infection [5]. In addition, host characteristics could contribute to vulnerability to neurologic complications related to influenza. Gika et al. [9] identified a mutation in the gene for Ran-binding protein 2, which has functions in nuclear import and membrane assembly, in both a 9-year-old Caucasian girl with recurrent influenza-associated acute necrotizing encephalopathy and her mother. Chen et al. [10] reported that Japanese patients with ther- 
molabile phenotype variations of carnitine palmitoyltransferase II, who expressed a disorder of mitochondrial $\beta$-oxidation, were prone to having encephalopathy following an influenza infection [10].

As mentioned above, CSF results are usually unremarkable in most patients with influenza-associated encephalitis/encephalopathy [2-7]. In a few patients with influenza-associated encephalitis/encephalopathy, mild pleocytosis, normal glycorrhachia, and a moderate elevation of CSF proteins $(45-100 \mathrm{mg} / \mathrm{dL})$ were the common findings [4]. In our patient, meningitis was also diagnosed based on nuchal rigidity and CSF findings. Mild pleocytosis and normal glucose levels in the CSF suggested that this patient had viral meningitis. However, markedly elevated levels of CSF protein are uncommon in viral meningitis. We cannot exclude the possibility of infections by other pathogens in this patient, even though all of the tests for microbiology were negative.

The diagnosis of influenza $\mathrm{A}$ infection in the patient reported here is probable based only on the positive nasopharyngeal swab rapid antigen test for influenza A. Both the viral cultures and the RT-PCR nasopharyngeal swab for influenza were negative. These negative results may have been caused by the 3-day treatment of osel- tamivir and the delayed examinations of viral culture and RT-PCR for influenza collected 9 days after the onset of symptoms.

\section{Conclusion}

Neurologic complications are infrequent in patients with influenza infection compared to their respiratory symptoms. We reported a 35-year-old female with influenza A infection presenting with acute respiratory distress syndrome, meningoencephalitis, and encephalopathy. Her brain MRI showed a single lesion of T2 prolongation in the left frontoparietal subcortical white matter that was consistent with focal encephalitis. This case report highlights a unique focal encephalitis found in brain MRI scans of a patient with concomitant severe pulmonary and neurologic complications after influenza infection.

\section{Disclosure Statement}

The authors report no conflict of interests.

\section{References}

1 World Health Organization: Influenza (seasonal). 2016. http://www.who.int/mediacentre/factsheets/fs211/en/ (accessed July 11, 2017)

2 Morishima T, Togashi T, Yokota S, et al: Encephalitis and encephalopathy associated with an influenza epidemic in Japan. Clin Infect Dis 2002;35:512-517.

3 Goenka A, Michael BD, Ledger E, et al: Neurological manifestations of influenza infection in children and adults: results of a $\mathrm{Na}-$ tional British Surveillance Study. Clin Infect Dis 2014;58:775-784.
4 Glaser CA, Winter K, DuBray K, et al: A population-based study of neurologic manifestations of severe influenza $\mathrm{A}(\mathrm{H} 1 \mathrm{~N} 1) \mathrm{pdm} 09$ in California. Clin Infect Dis 2012;55:514-520.

5 Omari I, Breuer O, Kerem E, et al: Neurological complications and pandemic influenza $\mathrm{A}$ (H1N1) virus infection. Acta Paediatr 2011; 100:e12-e16.

6 Farooq O, Faden HS, Cohen ME, et al: Neurologic complications of 2009 influenza-A H1N1 infection in children. J Child Neurol 2012;27:431-438.

7 Ekstrand JJ, Herbener A, Rawlings J, et al: Heightened neurologic complications in children with pandemic H1N1 influenza. Ann Neurol 2010;68:762-766.
8 Ishida $\mathrm{Y}$, Kawashima $\mathrm{H}$, Morichi S, et al: Brain magnetic resonance imaging in acute phase of pandemic influenza A (H1N1) 2009-associated encephalopathy in children. Neuropediatrics 2015;46:20-25.

9 Gika AD, Rich P, Gupta S, et al: Recurrent acute necrotizing encephalopathy following influenza $\mathrm{A}$ in a genetically predisposed family. Dev Med Child Neurol 2010;52:99-102.

10 Chen Y, Mizuguchi H, Yao D, et al: Thermolabile phenotype of carnitine palmitoyltransferase II variations as a predisposing factor for influenza-associated encephalopathy. FEBS Lett 2005;579:2040-2044. 